\title{
HOW TO MASTER MODULAR REALITIES IN ART, SCIENCE AND TECHNOLOGY — WITH NEW ELECTRONIC MEDIA, AND FOR A BETTER FUTURE OF WORK
}

\author{
Peter Purg \\ University of Nova Gorica, School of Arts, Nova Gorica, Slovenia
}

\begin{abstract}
The MAST project is developing an applied study module at the intersections of Art, Science and Technology, combining methodologies and practices that intertwine the academic sphere closely with the Culture and Creative Sectors. Nurturing a critical perspective on the historical, economical, social and above all cultural relevance of this interdisciplinary blend within the new digital shift, MAST develops innovative, ICT-enhanced teaching and learning methods. Students from different countries and disciplines will, under mentorship of engineers, scientists and artists, in partnership with relevant NGOs and industry partners, jointly tackle challenges emerging from the paradox between the obviously disparate agendas of Europe's ambition towards innovation on the one side, and the need for social equity on the other. In the present contribution, besides discussing MAST's Visual Identity Design in Pedagogical Context as well as contextualizing Web-design and Production as a uniquely Transversal Academic Collaboration process, Social Media shall be presented in their potential of becoming a Collective Reflection Tool, whereas the electronic publishing potential of Open Courses on Interoperable Platforms will be delineated. These four key aspects will indicate a vision of sustaining the impact of new curricula for a fair digital future that may keep a dynamic balance between the (European, competitive) need for simultaneously sustaining innovation and social agendas. The first piloting year of MAST poses the overarching challenge about the future of work in the context of electronic media, and seeks to develop (in both students, professionals and academia) positive digital-domain worker profiles. Integrating new media and electronic publishing approaches, these workers are able to think about future independently and freely, in a bold transdisciplinary manner, and act ethically across Creative \& Cultural Sectors and high technologies.
\end{abstract}

Key words: electronic dissemination, social media, web-design, visual identity, open courseware, social agenda, innovation

\section{INTRODUCTION TO THE MAST PROJECT}

Currently in its first year, the project "Master Module in Art, Science and Technology" (MAST) is developing an applied study module at the intersections of art, science and technology, combining methodologies and practices that will intertwine the academic sphere closely with the industry realms of the Culture and Creative Sectors (CCS). Nurturing a competent perspective on the historical, economical, social and above all cultural relevance of this interdisciplinary blend within the new digital shift, the project will apply innovative, ICT-enhanced teaching and learning methods. Gradual work-placement combined with support for spin-out or start-up and career self-management will instigate such innovation capacities in both students and the academy that are linked to the real and foreseeable CCS industry needs, but still critically reflect (its) realities and paradoxes. MAST is already in 2018/19 offering a range of five international workshops (Portugal, Austria, Hungary, Croatia, Slovenia), where students from three universities will face an interdisciplinary challenge, seeking innovative solutions with support of excellent mentors, artists, scientists, engineers, curators and producers.

However, to enable a most positive collision of the three realms, the project will need to let creatively collide (and combine, if not blend) the tech-innovation paradigm and the social equity agenda models. Technologies are always political and have a social impact, in that they codify certain values into material culture thus enabling (or limiting) individual and societal possibilities. Therefore, hard-won values - like social justice, strong labor, gender equality, and quality culture - will be linked with new digital innovations. In its tectonic ethical orientation, the module shall explore how progressive Social Europe (SE) agenda values can be coded into innovations, as well as how social groups and movements may use media and (high) technology to forward the values of SE. Experts from different fields will learn how to understand artists (i.e. their poetical, metaphysical, philosophical and ethical premises) and translate these divergent ideas into possible solutions that may reach all the way from industry-oriented innovative technologies to social innovation. 
This GRID 2018 contribution may be considered especially relevant and interesting in the "Electronic publishing and new media" context due to the MAST consortium agreeing on a method that integrates the pedagogic process with the visual identity and website development of the project (BA students taking over the responsibility, under deep involvement of internal and external mentors). Besides discussing Visual Identity Design in Pedagogical Context and contextualizing Web-design and Production as a uniquely Academic Collaboration process, the Social Media use shall be sketched in its potential of becoming a Collective Reflection Tool, along with a presentation of the electronic publishing potential of Open Courses on Interoperable Platforms. These four key aspects will delineate a vision of sustaining the impact of new curricula for a fair digital future that may keep a dynamic balance between the (European, competitive) need for innovation on the one hand and social agendas on the other.

\section{THE CHALLENGE BETWEEN INNOVATION AND SOCIAL AGENDAS (BACKGROUND)}

For Europe to successfully remodel its economy in a global competition, it must re-imagine innovation on -European terms. MAST therefore attempts to create sea $t$ of teaching and learning (as well as collaboration) methods by which the art, science, and technology crossover can be embedded with SE values, regardless of the particular application domain. Technologies are always political and have a social impact, in that they codify certain values into material culture, thus enabling (or limiting) individual and societal possibilities (c.f. the current perspective on creative value chains in "Mapping the Creative Value Chains executive summary by the European Commission (2017)). In this sense, technologies are a form of legislation outside the traditional space of politics: they are rarely democratic, transparent, or even acknowledged. Science is currently being productively challenged by participatory practices of citizen science and coproduction, and thus MAST represents a hybrid approach combining principles from art, design, sociology, politics, and other key Social Sciences and Humanities disciplines, as well as engineering and technology.

Surpassing the 'Silicon-Valley' modeled technical culture that in its deep structures principally opposes the social cohesion in both the national and local, as well as a global sense, MAST promotes a clear European vision as well as academic practice that aims to succeed in integrating the values of Social Europe into the entire chain of technology creation as well as use (including education). Studies agree (European Comission, 2017) that Europe is hamstrung by the tension between regressive technological ideology and what this project considers to be essential European social values of its ICT practitioners and their communities. The MAST graduates as European ICT, creative media and applied arts or design creators will need to be able to consider the social costs within a design as readily as they can do that with power, efficiency or the ergonomic aspects of a design or prototype. Over 90\% of Europe's search market belongs to Google, with the leftovers divided up between mostly non-European enterprises ("Here's How Dominant Google Is In Europe" (Rosoff, 2014)) and this lack is increasingly echoed for mobile platforms, browsers, etc. but even worse for whole genres of product ideas! Nevertheless, MAST embraces the fact that particularly in the Art+Sci+Tech crossover, there are key Soft skills that can only be developed on higher education level through well-designed training, in order to achieve positive social progression \& sustainability (STEPS, 2014).

Many of the most important production tools for digital media artists today are not the results of forprofit companies, but collaboratively designed using the ascending methods of free/open non-proprietary software and other technologies, as well as horizontal coordination of work and management models. Art and design training contributes to business, while art and commerce have always had a productive tension. The MAST consortium does ignore this tension, but rather to address it fully. This includes not only teaching business culture, but more broadly organizational techniques, which may be expressed in many forms. In particular, the project will seek to explore the ways that art, science, and technology crossover may be socially impactful in a variety of enterprise organizational forms, including business, non-profit, free/open software, platform collaborative, and others. MAST thus develops the intellectual framework and concrete repertoire by which responsible research and innovation can engender ICTs that strengthen the best qualities of the European social model. It does this by developing an educational module on HE level that corresponds to all relevant educational quality criteria (Quality and relevance in higher education (European Comission, 2018a). With it the graduate students of the MAST module will be in capacity to build an overall better electronic (publishing, communication, business etc.) world; their coworkers can have better jobs, and their users or customers can have products and services that do not undermine their culture, their way of (quality) life. 


\section{NEW APPROACHES TO ELECTRONIC PUBLISHING (METHODOLOGICAL DISCUSSION)}

The dissemination activities lie at the core of the MAST project and are focused on outreach actions that will spread the knowledge and collaboration outside of the consortium and partners directly involved in the project. Such actions will deepen collaboration and attract members of the industries towards potential collaboration on future educational activities and career development. The key objectives are to make the target groups aware of the MAST project, and keep following its messages as well as key (interim) results; to provide all needed mechanisms and an electronic platform (website, newsletter and social media) that informs the target groups about the project; and not least to build MAST as a brand that the target groups can associate with and use in their own communication as well as multiply accordingly for exploitation reasons, to eventually broaden the project's impact not only horizontally (taking over of models and practices) but also vertically (policy and business impact).

\subsection{Visual Identity Design in Pedagogical Context}

To reflect the academic as well as social and not least economic values and production principles argued for above, the visual Identity of the MAST project was conceived in collaboration with students, who took over the leading role in graphically designing the logotype and the outlines of the project's visual communication. A student who responded to an open invitation at the School of Arts, University of Nova Gorica, and was chosen for the task, accepted the first brief and discussed the visual identity at the project's kickoff meeting with the representatives of the project consortium (in which two partner institutions are explicitly concerned with dissemination activities).

The logotype of the project has been gradually developed subsequently by including both lexemic (letter $\mathrm{M}$ for MAST) as well as semantic elements (drop as a graphical element of most diverse possible meanings, denoting both natural sciences as well as the fluid character) of the project. Eventually a project-internal Visual Identity Handbook has been developed, with a special consideration of its use in digital realms (black-and-white, optimized low-resolution usage). The student involved in the development of the logotype is foreseen to take part in the piloting of the MAST module as such, in order to better reflect its identity and iterate own development of graphic design skills. The social (collective) reflection of the developed logotype was a key factor in its development, for which collaborative (Google) online documents were used in order to assure everyone's access not only to the development files in real-time, but also to give possibility of relevant, structured feedback, the tracking of versions etc.

\subsection{Web design and Production as a Transversal Academic Collaboration}

The web-design process was integrated into the pedagogical process even much deeper than the graphic design of the visual identity. Together with the undersigned project coordinator, the lecturer of webdesign in the first year of the undergraduate programme (Digital Arts and Practices) developed a stratified task, in which the students gradually developed possible wireframes for the project website, therein considering the possible semantic and symbolic interactions of the art-science-technology fields. Simultaneously they were discussing (and applying) the abovementioned Visual Identity, with which they developed a mutual interdependence (both were developed at the same time). Their guest mentor, also a renowned media artist, became the main programmer and webmaster to the MAST site.

After several sessions the most convincing website designs were chosen (by a mixed group of mentors and peers), upon which a most compatible Wordpress web-design theme was chosen and purchased, also reflecting the functional needs of the educational context that the project is primarily embedded in. The most interested and at the same time skilful student was chosen to collaborate on preparatory activities for the development of the theme into the actual website, upon which the below signed project coordinator and the PR (communications) officer of the project gradually filled in the contents of the website. At the time of writing the present contribution, the website launch at www.mastmodule.eu (MAST Consortium, 2018) is shortly due, and the social media presence is to be gradually developed on public level. A revision and consolidation of the electronic public relations (publications) strategy in the light of the recent project developments is planned within the following weeks. 


\subsection{Social Media as a Collective Reflection Tool}

The public events of the project that will have an impact not only on the academic communities involved, but also on the broader environment, consist of intensive learning events and so called pop-up events, as well as an annual "Interfacing Academy" event for showcasing and evaluation of the developed innovations, as well as the academic module as a whole. These events shall be promoted and disseminated publically via the project website and the related social media (Twitter, Facebook and Instagram profiles of MAST) that will all involve relevant stakeholders from in and around the MAST community, as well as externals. They shall involve a broad variety of stakeholders, including students, artists, engineers, scientists, guest lecturers, policy makers and industry representatives. The dissemination of central messages of the project will be delivered through a regular newsletter, sent out to a gradually assembled list of relevant e-mail addresses as well as featured on the website and promoted through relevant social media.

The social media activity will be designed and coordinated (moderated) in a way to stimulate feedback that will be sought both about the structure and contents of the module's curriculum, as well as the quality and relevance of the (prototyped, or speculatively designed) innovations that the student groups will develop during the academic cycle. The data will be eventually used for evaluation the project and fine-tuning the structures of the curriculum (courses, assessment forms, methodologies, inter-course connections etc.).

\subsection{Open Courses on Interoperable Platforms}

The success of initiatives such as MAST depends on an effective knowledge sharing strategy. This strategy will consider five areas of emphasis: organizational context, interpersonal and team characteristics, cultural characteristics, individual characteristics, and motivational factors. Each of these dimensions needs to be approached with a different method, but in an integrated fashion considering its interdependencies. Each consortium partner will contribute with different perspectives (such as industry, research, arts and science) on the different components of MAST (curriculum, mobility, partnering strategies, teaching methodologies and disciplines) to create a best practices book that will outline a collaboration framework for Art, Science and Technology and best practices featured therein. It is to be available publically in the form of an e-book via a web service, at no cost.

The consortium shall develop a so called 'cross-sector innovation transfer' business plan, based on online media. This integrated format shall both deepen and widen the impacts of MAST, including long-term sustainability, the main focus being on how to assure a lasting continuation and/or legacy of the project activities. This activity shall also develop a knowledge transfer model through a free of charge (open) online course, with a wide variety in both artistic and technical content, involving experts from various fields. The course shall equip participants with digital, entrepreneurial and design/art knowledge and thus foster students from art and cultural institutions to acquire interdisciplinary skills and experiences with emerging technologies. Even if designed as a widely offered online course, it shall be used also in the entry stages of the MAST module piloting, in the second academic cycle. The online seminar shall be accompanied by a digital forum, possibly embedded into social media such as Facebook or Twitter, where students of any related field could share their questions, experiences and discuss the lessons learned with both peers and professionals, curators, entrepreneurs and artists from the field(s).

\section{SUSTAINING THE IMPACT OF NEW CURRICULA FOR A FAIR DIGITAL FUTURE (CONCLUSIONS AND OUTLOOK)}

The above methods and approaches indicate at how the MAST project attempts to use new media and digital publishing tools to disseminate and evaluate the project's activities and products. It still remains to be validated whether these approaches shall actually serve the purpose planned, however it may be argued already that these tools and methods do reflect the contemporary realities of communication and collaboration found in the interdisciplinary field of art, science and technology. Moreover, they also bring about important topical implications, such as the current academic year's challenge to the students: 'How can work, collectives, or enterprises be designed to ensure a more inclusive, supportive, verdant, and open society?' Along the way of developing efficient solutions to meaningful challenges in the realm of technological innovation, the MAST community should explore how key choices in art, design, and technology can help or harm a virtuous circle of progressive European social values. Among many other policies, documents, proclamations and practices on both European and national as well as local and non- 
governmental levels, these values are well reflected in the current European Pillar of Social Rights (European Commission, 2018b) that is about delivering new and more effective work-related rights for citizens, built upon 20 key principles along the chapter of Equal opportunities and access to the labor market; Fair working conditions; as well as Social protection and inclusion. The underlying assumption so far is that the future of (electronically supported, digitally dominated) work should belong to (or at least be championed by) profiles who are able to think about future independently and freely, in transdisciplinary manner, inserting and transforming existing solutions and products into new scenarios that would be solved or transferred to industry realms, ranging from CCS to high technologies and social services. The ideal MAST graduate should act as a coordinator and an integrator in these realms, remaining in positive (if not utterly creative) control over her or his (and our common) digital tools and electronic platforms.

\section{ACKNOWLEDGMENTS}

This contribution rests on the project conception and legacy of the MAST project, conceived by the MAST consortium and supported by the European Commission (Communications Networks, Content \& Technology; CONNECTI.3 Unit - Audiovisual Industry \& MEDIA programme), where it is necessary to note that the content of this contribution reflects the views only of the author(s, including the MAST consortium), and the European Commission cannot be held responsible for any use which may be made of the information contained therein. The MAST consortium combines a host of hi-profile institutions with excellent references across the science-art-technology triangle. Partner roles in the MAST consortium typically combine academic, managerial and professional (business) profiles with two institutions of big outreach capacity, as well as several topically relevant associate partners: University of Nova Gorica, School of Arts (Slovenia) in the managerial (consortium coordination) role is joined by Madeira Interactive Technologies Institute (Portugal) and Technical University Graz, Institute of Spatial Design (Austria) in academic as well as research \& development roles. Kersnikova Institute (Slovenia) and Kitchen Budapest (Hungary) represent the entrepreneurial production partners, while networking and outreach are covered by Culture Action Europe (Belgium/EU) and the Croatian Cultural Alliance / Unicult programme (Croatia). Associated partners are: EQ-Arts (Netherlands); University of Madeira - UMa(Portugal); Stromatolite (Sweden/UK); The University of Arts Belgrade (Serbia); Institute for Development and International Relations - IRMO (Croatia); Hakan Lidbo Audio Industries (Sweden); European Creative Business Network - ECBN (EU wide); European Digital Art and Science Network (EU wide).

\section{REFERENCES}

[1] European Commission: "Mapping the Creative Value Chains. A study on the economy of culture in the digital age. Executive Summary", 2017, URL https://publications.europa.eu/en/publicationdetail/-/publication/4737f41d-45ac-11e7-aea8-01aa75ed71a1/language-en (last request: 2018-09-28).

[2] STEPS: "Soft Skills; Training; Enabling; Progression \& Sustainability", 2014, URL s3.amazonaws.com/2seas-us/page_ext_attachments/1685/STEPS_MAG_Nov2014_EN.pdf (last request: 2018-09-28).

[3] European Commission: "Quality and relevance in higher education", 2018a, URL http://ec.europa.eu/education/policy/higher-education/quality-relevance_en (last request: 2018-09-28).

[4] Rosoff, M.: "Here's How Dominant Google Is In Europe". Business Insider, 2014, URL http://www.businessinsider.com/heres-how-dominant-google-is-in-europe-2014-11 (last request: 2018-09-28).

[5] MAST Consortium: "Mast Module", URL www.mastmodule.eu (last request: 2018-10-07).

[6] European Commission: "European Pillar of Social Rights", 2018b, URL https://ec.europa.eu/commission/priorities/deeper-and-fairer-economic-and-monetaryunion/european-pillar-social-rights/european-pillar-social-rights-20-principles_en (last request: 2018-10-07). 


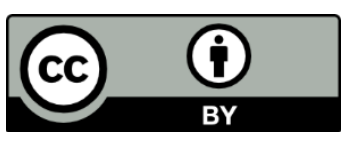

(C) 2018 Authors. Published by the University of Novi Sad, Faculty of Technical Sciences, Department of Graphic Engineering and Design. This article is an open access article distributed under the terms and conditions of the Creative Commons Attribution license 3.0 Serbia

(http://creativecommons.org/licenses/by/3.0/rs/). 